\title{
Modelos conceptuales de abundancia de fitoplancton asociados a la heterogeneidad espacial en el Embalse Rapel (Chile central)
}

\author{
Conceptual models of phytoplankton abundance associated to spatial heterogeneity at \\ the Rapel reservoir (central Chile)
}

GINGER MARTÍNEZ ${ }^{1,2}$, MANUEL CONTRERAS ${ }^{1,2} \&$ IRMA VILA $^{1}$

\author{
${ }^{1}$ Laboratorio de Limnología, Departamento de Ciencias Ecológicas, Facultad de Ciencias, Universidad \\ de Chile, Casilla 653, Santiago, Chile; e-mail: gingerma@cec.uchile.cl \\ ${ }^{2}$ Centro de Ecología Aplicada Limitada, Avenida Suecia 3304, Santiago, Chile
}

\begin{abstract}
RESUMEN
La heterogénea morfología de los sistemas acuáticos regulados genera patrones de abundancia y gatilla una respuesta diferencial de los ensambles planctónicos. Un ejemplo de ello ocurre en el Embalse Rapel (34¹0' S, 71²9’ O). Un gradiente morfoedáfico longitudinal y condiciones hidrodinámicas localizadas producen complejidad espacial en esta cuenca dendrítica, lo que ha sido propuesto como probable factor causal de las diferencias de abundancia fitoplanctónica entre distintos sectores del embalse. En el presente estudio se proponen mecanismos y modelos conceptuales de abundancia de fitoplancton y para ello se describieron patrones de distribución de algas en tres sectores del embalse: Las Balsas, Alhué y El Muro. Posteriormente se identificaron las principales variables predictoras de abundancia fitoplanctónica y se estableció un modelo de funcionamiento específico para cada sector del embalse. A partir de una base de datos bibliográficos se obtuvo información de abundancia total de fitoplancton y de variables físicas y químicas desde cuatro estaciones de muestreo representativas de cada sector además del sector de Confluencia entre los sectores Las Balsas y Alhué. Se realizaron análisis de clasificación jerárquica y de componentes principales para la descripción del patrón espacial y a través de un análisis de regresión múltiple se identificaron las variables predictoras en cada estación de muestreo. Los resultados mostraron dos agrupamientos significativos: $1=$ Las Balsas-Muro y $2=$ AlhuéConfluencia. Las variables físicas y químicas también se segregaron espacialmente en función de las estaciones de muestreo. Se detectaron diferentes conjuntos de variables predictoras en cada sector del embalse. En la estación Las Balsas, las variables asociadas a la abundancia de fitoplancton fueron la temperatura, concentración de fósforo soluble y conductividad específica. Por el contrario, en las estaciones Alhué y El Muro, la mayor variabilidad fue explicada por la concentración de compuestos nitrogenados, siendo el nitrato particularmente importante en el sector Alhué y el amonio en el sector El Muro. Estos resultados sugieren un control diferencial de la abundancia fitoplanctónica asociada a los diferentes sectores del embalse. Mientras que en el sector Las Balsas predominaría un control externo generado por la descarga de los tributarios, en la estación El Muro se postula un control interno y producido por la generación in situ de amonio. La significativa asociación a nitrato y nitrógeno orgánico detectada en la estación Alhué indicaría un control doble en la generación del patrón fitoplanctónico.
\end{abstract}

Palabras clave: heterogeneidad, patrones espaciales, fitoplancton, embalse.

\begin{abstract}
Heterogeneous morphology generates abundance patterns and a differential response of the planktonic assemblages in regulated aquatic systems. This is the case at the Rapel reservoir ( $\left.34^{\circ} 10^{\prime} \mathrm{S}, 71^{\circ} 29^{\prime} \mathrm{W}\right)$. A longitudinal morphoedaphic gradient and localized hydrodynamic conditions produce spatial complexity in this type-dendritic basin, that has been proposed as probable causal mechanism of the main diferences in phytoplankton abundance among areas inside of the reservoir. In this study, mechanisms and conceptual models of phytoplankton abundance are proposed, describing algae distribution patterns in three reservoir areas: Las Balsas, Alhué and El Muro. Also, the main predictive variables were identified which lead to propose a specific functioning model in each reservoir area. A data base published was used in order to obtain phytoplankton total abundance and physical and chemical variables from each station sampling that represent the reservoir areas, including the Confluencia area localized between Las Balsas and El Muro areas. Cluster and principal components analysis were applied in order to describe the spatial pattern and a multiple linear regression analysis was utilized to identify predictive variables in each sampling station. Results showed two significant groups: 1 = Las Balsas-El Muro, and 2 = Alhué-Confluencia. Physical and chemical variables also were segregated in the space, according to the sampling stations. Different groups of predictive variables among the stations were found. At the Las Balsas station, variables associated to phytoplankton abundance were temperature, soluble reactive phosphorous concentration and specific conductivity. On the contrary, at the Alhué and El Muro stations, the greater variability was explained by the nitrogen compounds concentration, and nitrate specially important at the Alhué station and ammonia at the El Muro station. These results suggest a differential control of the phytoplankton abundance
\end{abstract}


associated to each reservoir areas. While in Las Balsas station an external control generated by discharge of tributaries would prevail, in the El Muro station an internal control is proposed, that emerges from the ammonia in situ generation. At the Alhué station, the significant association to nitrate and organic nitrogenous would reveal a double control in the generation of the phytoplanktonic pattern.

Key words: heterogeneity, spatial patterns, phytoplankton, reservoir.

\section{INTRODUCCIÓN}

Desde un perspectiva espacial, los ecosistemas acuáticos representan escenarios en tres dimensiones, en donde la heterogeneidad espacial del medio genera patrones de distribución en los ensambles planctónicos (Fernández et al. 1994, Pourriot et al. 1994, Armengol et al. 1999, Pérez et al. 1999). Atributos morfológicos, específicamente, el tamaño, volumen y área de la cubeta han sido considerados predictores significativos de la producción, composición y predominio de especies (Bahamonde \& Cabrera 1984, Tundisi et al. 1999). En sistemas regulados, el principal eje de variación morfológica lo constituye un gradiente de condiciones morfoedáficas, el que se desarrolla longitudinalmente en dirección al muro de contención (Lind \& Dávalos-Lind 1999, Berrada et al. 2000, Espíndola et al. 2000). A través de este eje, una condicion lótica es progresivamente reemplazada por una condicion léntica conduciendo a la compartamentalización física del sistema (Thornton 1990). A una escala local, la incorporación de la descarga de tributarios y de la efluencia a través de la presa generan patrones de circulación vertical que aumentan la complejidad espacial del sistema (Tundisi 1993, Kennedy 1999, Romero \& Imberger 1999, Straskraba 1999, Kwang \& Jones 2000). Estos gradientes afectan la distribución de nutrientes para la biota y por lo tanto indirectamente ejercen un importante control sobre la distribución de las poblaciones planctónicas (Henry et al. 1998, Kawara et al. 1998, Tundisi et al. 1998, Padisák et al. 1999, Nogueira 2000). Un ejemplo de esta situación lo constituye el Embalse Rapel (Chile central). Numerosos trabajos señalan que los gradientes morfoedaficos serían la principal causa de los patrones de abundancia de fitoplancton observados en diferentes sectores de este embalse (Cabrera et al. 1977, Soto et al. 1984, Vila et al. 1987, Contreras 1992). La interacción entre la morfología lacustre, aportes fluviales y la acción de vientos predominantes produciría diferencias en la composición de fitoplancton (Reynolds et al. 1986, Vila et al. 1987), en los patrones de productividad primaria (Montecino \& Cabrera 1982) y en la condición trófica (Lavanderos et al. 1994, Vila et al. 2000) del Embalse Rapel.
Complementando estos estudios, el análisis de imágenes satelitales en este emblalse ha revelado tres sectores en el plano horizontal, claramente definidos en función de la carga de sedimentos y de la concentración de clorofila a, patrón atribuido a las diferencias en las condiciones de circulación encontradas en el sistema (Lavanderos et al. 1994). Simulaciones desarrolladas en laboratorio han confirmado estos resultados e indican que la unidireccionalidad del flujo que se genera entre el sector de afluencia y la presa produciría un patrón de circulación heterogéneo y el aislamiento hidrodinámico de un sector del embalse Rapel (Hillmer \& Niño 2000¹). También, este aislamiento respondería a diferencias de caudales que aportan hacia cada uno de los sectores, en estrecha relación con el régimen de vientos predominantes y la morfología de la cubeta (Antenucci 1996). Por lo tanto, la alta variabilidad espacial que representa el gradiente morfoedáfico y las diferencias en hidrodinámica desarrolladas localmente en el Embalse Rapel, han conducido a plantear una respuesta diferencial del ensamble de fitoplancton y patrones de distribución diferentes entre distintos sectores del embalse.

En el siguiente estudio se propone describir el patrón de distribución espacial de la abundancia total de fitoplancton e identificar las principales variables que generarían tal patrón. A partir del análisis se postulan tres modelos conceptuales que pudieran explicar la distribución de la abundancia de fitoplancton en el embalse Rapel.

\section{MATERIALES Y MÉTODOS}

\section{Área de estudio}

El Embalse Rapel (34¹0’ S, 71²9’ O) está ubicado en la zona central de Chile, a $140 \mathrm{~km}$ al suroeste de la ciudad de Santiago (Chile) (Fig. 1). Este

${ }^{1}$ HILLMER I \& Y NIÑO (2000) Análisis de la hidrodinámica del Embalse Rapel y sus consecuencias en el transporte y mezcla de contaminantes. Taller 'Cuenca Rapel: diagnóstico, evaluación y líneas de acción'. Codelco-Chile, Santiago, Chile. 


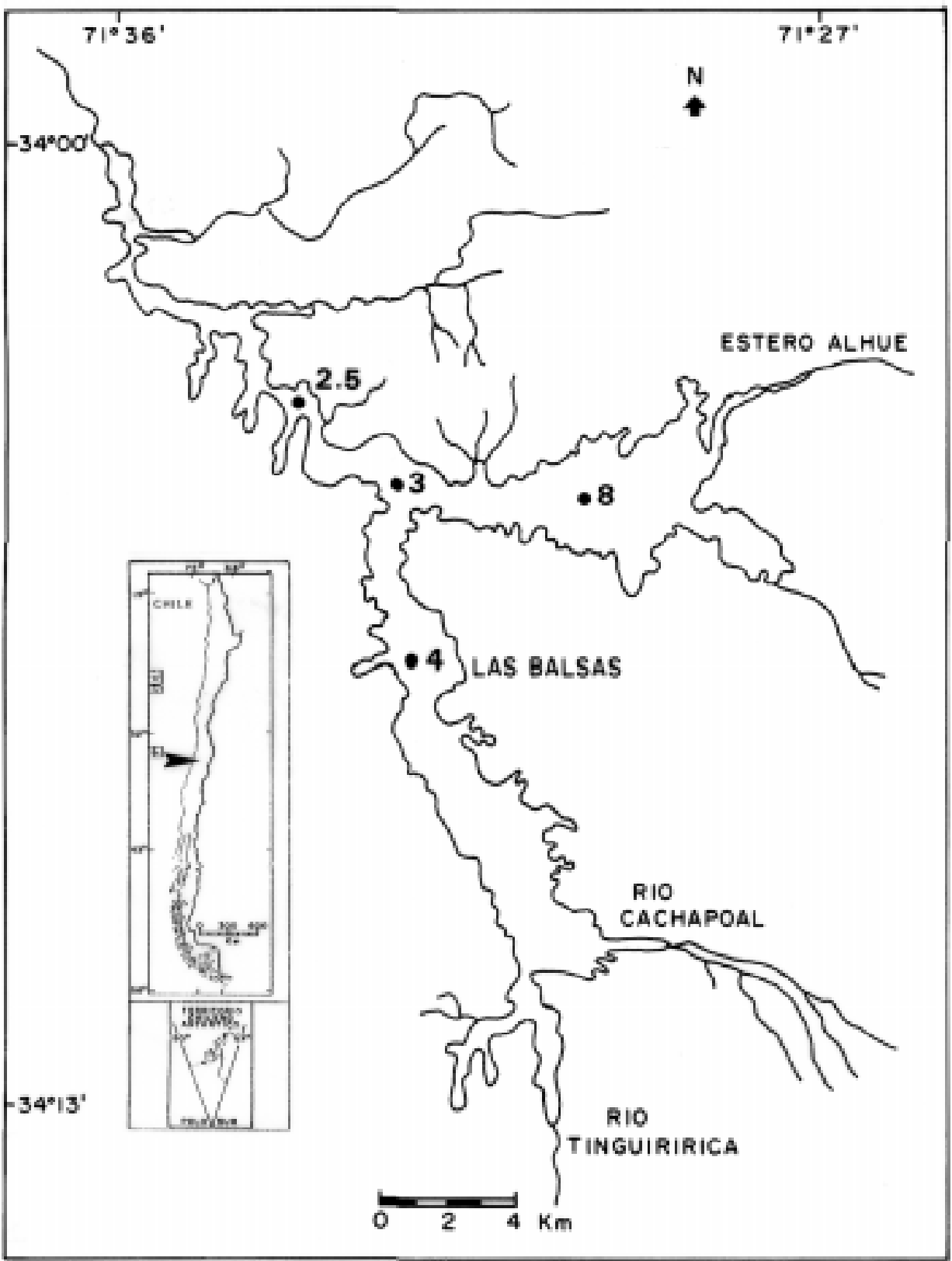

Fig.1: Ubicación de las estaciones de muestreo de parámetros físicos y químicos y de fitoplancton en el Embalse Rapel (Chile central, $34^{\circ} \mathrm{S}, 71^{\circ} \mathrm{O}$ ).

Location of the sampling stations of physical and chemical parameters and phytoplankton at the Rapel reservoir (central Chile, $\left.34^{\circ} \mathrm{S}, 71^{\circ} \mathrm{W}\right)$.

embalse fue creado en 1968 y abastece de energía eléctrica al sistema interconectado central durante los periodos de otoño e invierno. El embalse representa un sistema monomíctico templado y muestra una compleja morfología asociada a una configuración de tipo dendrítica y a un alto desarrollo litoral (Contreras et al. 1994). Dentro del Embalse Rapel se han descrito tres sectores con significativas variaciones en las condiciones hidrodinámicas
(Contreras et al. 1994, Antenucci 1996, Hillmer \& Niño $2000^{1}$ ). Uno de ellos es el sector Las Balsas, ubicado en dirección $\mathrm{S}$ y que se inserta en el antiguo cauce del río Rapel. Este sector recibe la descarga de los principales tributarios del embalse, los ríos Cachapoal y Tinguiririca de régimen pluvio-nival. En dirección noroeste se localiza el sector de la presa, cuya base se ubica a $20 \mathrm{~m}$ de altitud. Este sector presenta la mayor profundidad 
del embalse (75 m, ver Tabla 1) y es afectado por la descarga del efluente. El sector Alhué está ubicado hacia el NE y se sitúa en el canal del estero del mismo nombre, principal tributario que descarga en este lugar. El sector Alhué muestra una condición somera y un alto grado de exposición a vientos predominantes (Contreras et al. 1994). Antecedentes de morfometría para cada uno de los sectores se presentan en la Tabla 1 .

\section{Procedimiento analítico}

El estudio se realizó con datos bibliográficos obtenidos en el embalse Rapel durante el periodo 1991-1992 (Proyecto FONDECYT 1074-92, Universidad de Chile). Se seleccionaron cuatro estaciones de muestreo, representativas de los principales sectores del embalse: (1) estación El Muro, ubicada próxima al sector de la presa, (2) estación Las Balsas, en el sector de afluencia de tributarios y (3) estación Alhué, sector con condiciones hidrodinámicas singulares dentro del embalse y ubicado en un tramo medio de la cubeta. También se incluyó la intersección de los sectores Las Balsas y Alhué, denominada estación Confluencia, ello debido a que muestra una condición intermedia entre ambos sectores (Fig. 1).

Los parámetros físicos considerados como variables forzantes fueron la temperatura, $\mathrm{pH}$ y conductividad específica (CE), los que fueron medidos in situ según lo recomendado por APHA (1995). Los parámetros químicos fueron oxígeno disuelto y compuestos nitrogenados y fosforados orgánicos e inorgánicos. La determinación de oxígeno disuelto se realizó a través del método
Winkler. La concentración de fósforo reactivo soluble $\left(\mathrm{PO}_{4}-\mathrm{P}\right)$ y de compuestos inorgánicos nitrogenados $\left(\mathrm{NO}_{2}-\mathrm{N}, \mathrm{NO}_{3}-\mathrm{N}, \mathrm{NH}_{4}-\mathrm{N}\right)$ fue medida según la metodología propuesta por Zahradnik (1981). La concentración de fósforo $\left(\mathrm{P}_{\text {тот }}-\mathrm{P}\right)$ y nitrógeno $\left(\mathrm{N}_{\text {тот }}-\mathrm{N}\right)$ orgánico total fue determinada según la metodología propuesta por Mühlhauser et al. (1987). Se utilizaron valores promediados en profundidad suponiendo homogeneidad térmica en la columna de agua. Simultáneamente se obtuvieron valores promediados de abundancia total de fitoplancton (unidades estándares $\mathrm{L}^{-1}$ ) desde las mismas estaciones de muestreo y que representó la variable de estado del sistema.

Se exploraron relaciones espaciales entre las variables mediante análisis de clasificación jerárquica basados en distancia euclidiana y de componentes principales (ACP) (Hair et al. 1995). Los agrupamientos que emergieron fueron sometidos a un análisis de varianza para medidas repetidas en el tiempo (Gurevitch \& Chester 1986) debido a que se incluyeron estaciones en diferentes épocas del año. Los dendrogramas fueron construidos utilizando el programa computacional SYSTAT (Wilkinson et al. 1992). Las principales variables predictoras de la abundancia total de fitoplancton fueron seleccionadas a través de un análisis de regresión múltiple y utilizando una estrategia backwards de eliminación de variables. Fueron removidas aquellas variables que presentaban un menor aporte explicativo, hasta disminuir en un $10 \%$ la adecuación inicial del modelo (Mardia et al. 1995). También se consideró como criterio de selección una distribución al azar de los residuos y la ausencia de multicolinealidad entre variables independientes.

TABLA 1

Parámetros morfométricos de tres sectores del Embalse Rapel. Datos extraídos de Contreras et al. (1994)

Morphometric parameters of three areas at the Rapel reservoir. Data obtained from Contreras et al. (1994)

\begin{tabular}{lcccc}
\hline Parámetro & Unidad & Alhué & Las Balsas & El Muro \\
\hline Área $(A)$ & $\mathrm{km}^{2}$ & 27,4 & 37,4 & 14,8 \\
Volumen $(\mathrm{V})$ & $\mathrm{km}^{3}$ & 0,115 & 0,210 & 0,342 \\
Perímetro $(\mathrm{P})$ & $\mathrm{km}$ & 57,4 & 93,6 & 72,8 \\
Profundidad máxima $\left(\mathrm{H}_{\max }\right)$ & $\mathrm{m}$ & 17,0 & 24,0 & 75,0 \\
Profundidad media $(\mathrm{H})$ & $\mathrm{m}$ & 4,2 & 5,6 & 19,2 \\
Ancho máximo $\left(\mathrm{A}_{\mathrm{m}}\right)$ & $\mathrm{km}$ & 5,6 & 3,6 & 3,5 \\
Ancho medio $\left(\mathrm{A}_{\mathrm{me}}\right)$ & $\mathrm{km}$ & 2,7 & 17,1 & 5,8 \\
Longitud máxima $(\mathrm{L})$ & $\mathrm{km}$ & 10,1 & 0,23 & 0,26 \\
Desarrollo de volumen $\left(\mathrm{D}_{\mathrm{v}}\right)$ & - & 0,25 & 4,3 & 4,9 \\
Desarrollo línea de costa $\left(\mathrm{D}_{\mathrm{L}}\right)$ & - & 3,1 & 12,4 & 12,4 \\
Tiempo de residencia $\left(\tau_{\mathrm{w}}\right)$ & $\mathrm{d}^{-1}$ & 1,2 & & \\
\hline
\end{tabular}




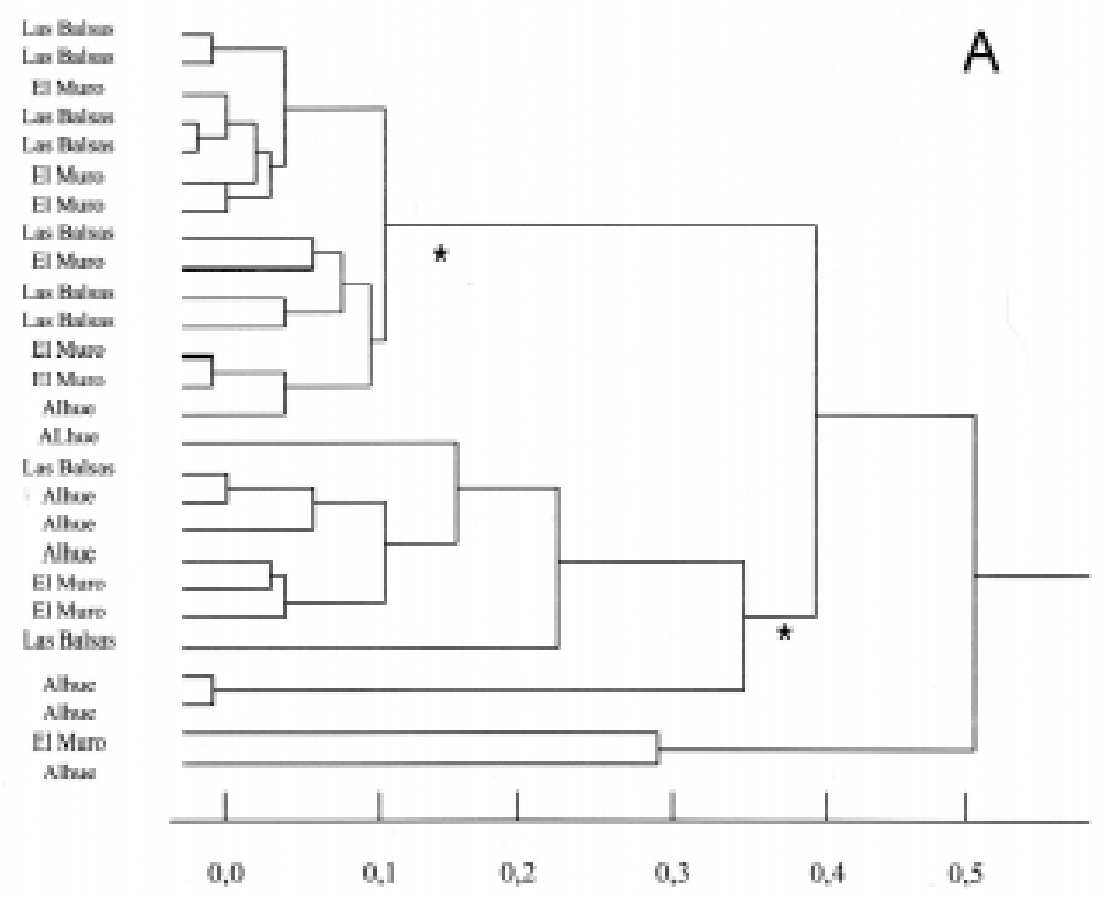

Distancia
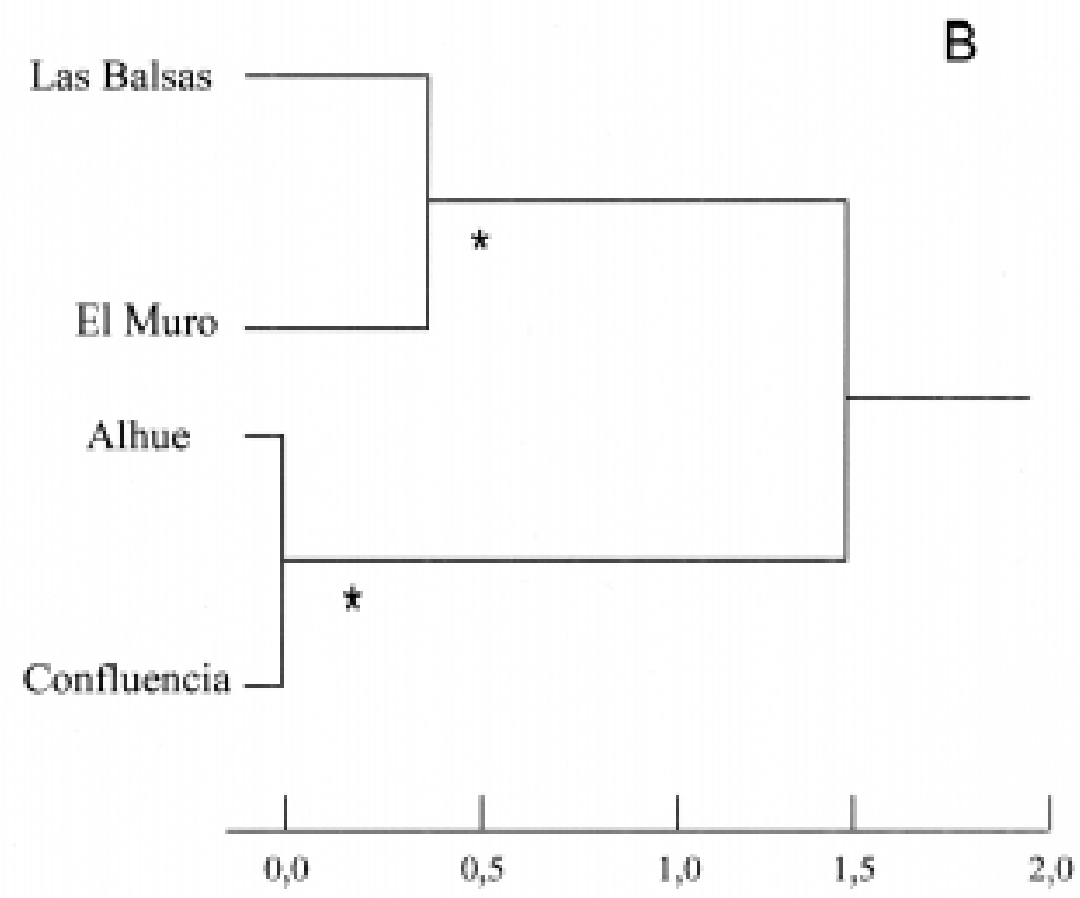

\section{Distancia}

Fig. 2: Análisis de clasificación jerárquica de la abundancia total de fitoplancton entre diferentes estaciones de muestreo, sin (A) y con (B) la estación de muestreo Confluencia: $(*)=\mathrm{P}<0,05$.

Cluster analysis of the phytoplankton total abundance among different sampling stations, without (A) and with (B) Confluencia sampling station: $(*)=\mathrm{P}<0.05$. 


\section{RESULTADOS}

El análisis de clasificación para la abundancia total de fitoplancton permitió separar dos grupos. El primer agrupamiento fue obtenido entre las estaciones Las Balsas y El Muro a una distancia de 0,1 (Fig. 2A). El segundo agrupamiento incluyó a la estación Alhué, a una distancia 0,35 (Fig. 2A). El análisis de varianza entre ambos grupos mostró que la media de la abundancia total del segundo grupo Alhué (1.183 $\mathrm{UE} \mathrm{L} \mathrm{L}^{-1}$, rango: 815.548) fue significativamente mayor a la media del primer grupo Las Balsas-El Muro (537 $\mathrm{UE} \mathrm{L}^{-1}$, rango: 32-3.360) (ANDEVA para medidas repetidas, $\mathrm{F}=84,212 ; \mathrm{P}=0,012$; Tabla 2). La inclusión de la estación Confluencia y los valores promediados en el tiempo confirmaron los dos agrupamientos obtenidos en el primer análisis (Fig. 2B). La estación Confluencia se asoció en forma significativa con la estación Alhué, a una distancia menor a 0,01 y el segundo grupo se mantuvo conformado por los sectores Las Balsas y El Muro a una distancia de 0,374 (Fig. 2B). El análisis espacial (ACP) realizado con variables físicas y químicas y adicionando un segundo sector Alhué (Alhué ${ }_{2}$ ) se presenta en la Fig. 3. Los resultados del ACP mostraron una segregación espacial significativa entre las cinco estaciones de muestreo, lo cual fue evidente entre el sector Alhué (estaciones Alhué ${ }_{1}$ y Alhué ${ }_{2}$ ) y el resto de las estaciones incluidas (Fig. 3). Los dos primeros factores explicaron un $83,6 \%$ de la variación total mostrada por el conjunto de variables físicas y químicas de cada estación de muestreo. El factor 1 explicó el $66,3 \%$ mientras que el factor 2 explicó un 17,3\%.

A través del análisis de regresión se detectaron diferentes variables predictoras de abundancia total de fitoplancton entre las estaciones de muestreo. En la estación Las Balsas, las variables asociadas a la abundancia total de fitoplancton fueron la temperatura $(\mathrm{T})$, la concentración de fósforo reactivo soluble $\left(\mathrm{PO}_{4}-\mathrm{P}\right)$ y la conductividad específica (C.E) (Tabla 3A). Estas variables contribuyeron en forma similar a explicar la abundancia total de fitoplancton, sin embargo, mostraron diferentes patrones de asociación. Mientras que la temperatura (coeficiente estandarizado $=-0,533$;

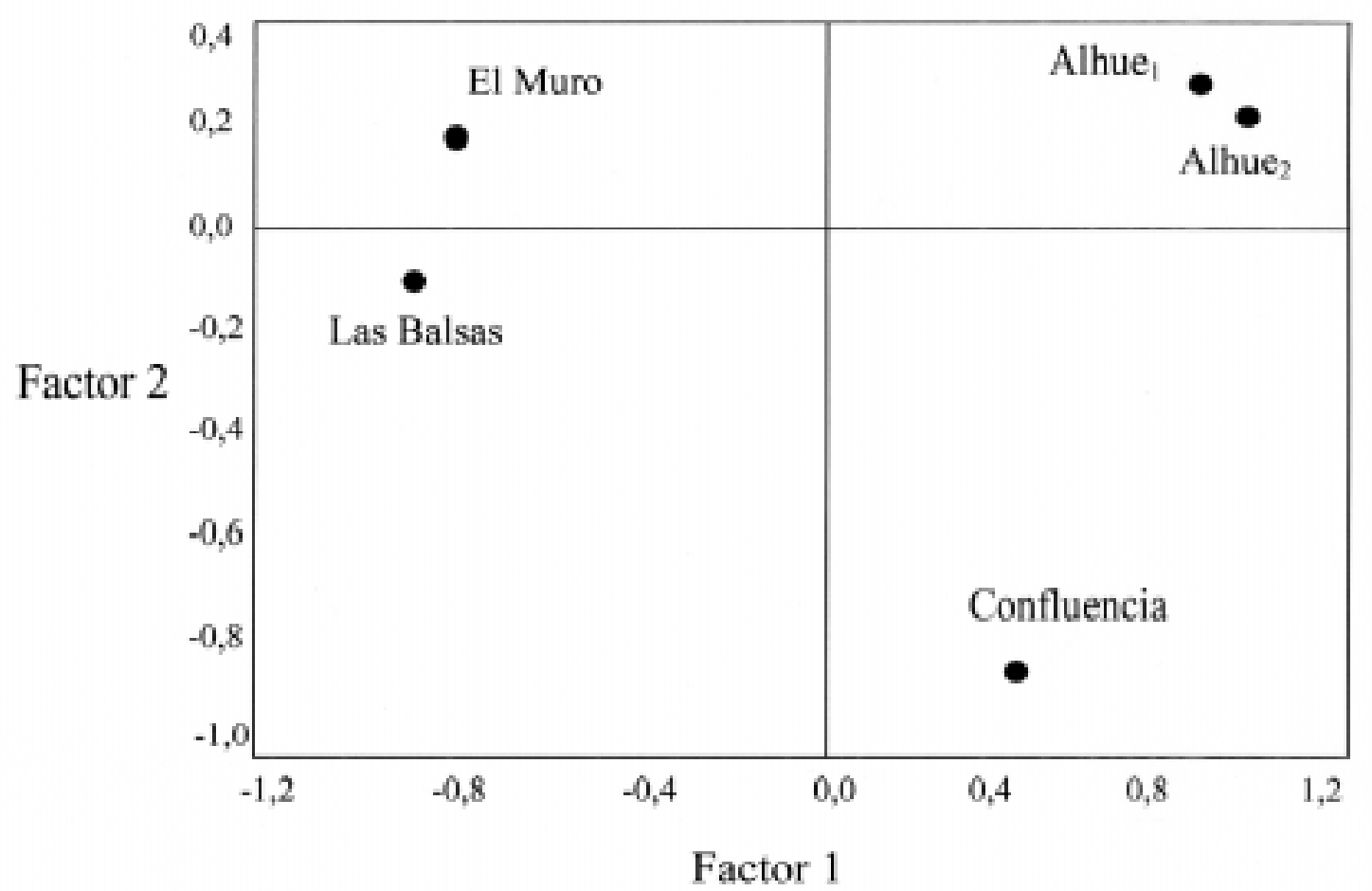

Fig. 3: Ordenamiento de sectores del Embalse Rapel mediante variables físicas (temperatura, pH, conductividad específica) y químicas (oxígeno disuelto, fósforo reactivo soluble, fósforo orgánico total, compuestos inorgánicos solubles y nitrógeno orgánico total) a través de análisis de componentes principales.

Ordination of Rapel reservoir areas through physical (temperature, $\mathrm{pH}$, specific conductivity) and chemicals (dissolved oxigen, soluble reactivate phosphorus, total organic phosphorus, soluble inorganic compounds and total organic nitrogenous) variables by principal components analysis. 


\section{TABLA 2}

ANDEVA de una vía para medidas repetidas de la abundancia total de especies de fitoplancton: $\mathrm{SC}=$ suma de cuadrados; $\mathrm{gl}=$ grados de libertad $; \mathrm{CM}=$ cuadrados medios; $\mathrm{P}=$ valor de probabilidad asociada al término de interacción entre las variables fitoplancton y tiempo

One-way repeated measures ANOVA of the phytoplankton total abundance: $\mathrm{SC}=$ sum of squares; $\mathrm{gl}=\mathrm{degrees}$ of freedom; $\mathrm{CM}=$ mean squares; $\mathrm{P}=$ probability value of the interaction term between phytoplankton and time variables

\begin{tabular}{lcccrc}
\hline Fuente de variación & SC & gl & CM & F & P \\
\hline Fitoplancton (F) & $2,36 \times 10^{6}$ & 1 & $2,36 \times 10^{6}$ & 84,212 & 0,012 \\
Tiempo (T) & $1,20 \times 10^{7}$ & 8 & $1,50 \times 10^{6}$ & 4,927 & 0,003 \\
FxT & $1,34 \times 10^{6}$ & 8 & $1,67 \times 10^{5}$ & 0,548 & 0,804 \\
Error $_{1}$ & $5,60 \times 10^{4}$ & 2 & $2,80 \times 10^{4}$ & & \\
Error $_{2}$ & $4,89 \times 10^{6}$ & 16 & $3,05 \times 10^{5}$ & & \\
\hline
\end{tabular}

TABLA 3

Variables predictoras de abundancia de fitoplancton en el Embalse Rapel. Resumen del análisis de regresión lineal múltiple entre variables físicas, químicas y la abundancia total de fitoplancton en tres estaciones de muestreo: (A) Las Balsas, (B) Alhué y (C) El Muro

Predictives variables of the phytoplankton abundance at the Rapel reservoir. Summary of the multiple linear regression analysis between physical, chemical and phytoplankton total abundance variables in three sampling stations: (A) Las Balsas, (B) Alhué, and (C) El Muro

(A) Estación de muestreo Las Balsas

$\mathrm{R}_{\text {Multiple }}=0,87 ; \mathrm{R}^{2}$ Multiple $=0,77 ; \mathrm{N}=21 ; \mathrm{F}_{(3,17)}=19,25 ; \mathrm{P}<0,001$

\begin{tabular}{lccccrr}
\hline Fuente de variación & Coeficiente & Error & $\begin{array}{c}\text { Coeficiente } \\
\text { estandarizado }\end{array}$ & Tolerancia & Valor de t & Valor de P \\
\hline Constante & $-4,596$ & 6,588 & 0,0 & - & $-0,697$ & 0,495 \\
Temperatura & $-1,979$ & 0,485 & $-0,533$ & 0,785 & $-4,083$ & $<0,001$ \\
PO $_{4}$-P & $-0,562$ & 0,160 & $-0,547$ & 0,550 & $-3,508$ & 0,003 \\
C. E. & 2,873 & 0,968 & 0,424 & 0,655 & 2,967 & 0,009
\end{tabular}

(B) Estación de muestreo Alhué

$\mathrm{R}_{\text {Multiple }}=0,63 ; \mathrm{R}^{2}{ }_{\text {Multiple }}=0,41 ; \mathrm{N}=21 ; \mathrm{F}_{(2,18)}=6,14 ; \mathrm{P}=0,009$

\begin{tabular}{lcccrrr}
\hline Fuente de variación & Coeficiente & Error & $\begin{array}{c}\text { Coeficiente } \\
\text { estandarizado }\end{array}$ & Tolerancia & Valor de t & Valor de P \\
\hline Constante & 5,281 & 1,464 & 0,0 & & 3,608 & 0,002 \\
$\mathrm{NO}_{3}$-N & $-0,241$ & 0,098 & $-0,448$ & 0,988 & $-2,451$ & 0,024 \\
$\mathrm{~N}_{\mathrm{TOT}^{-}} \mathrm{N}$ & 0,478 & 0,215 & 0,407 & 0,988 & 2,229 & 0,038
\end{tabular}

(C) Estación de muestreo El Muro

\begin{tabular}{|c|c|c|c|c|c|c|}
\hline Fuente de variación & Coeficiente & Error & $\begin{array}{l}\text { Coeficiente } \\
\text { estandarizado }\end{array}$ & Tolerancia & Valor de $\mathrm{t}$ & Valor de $\mathrm{P}$ \\
\hline Constante & 0,740 & 3,060 & 0,0 & & 0,242 & 0,811 \\
\hline $\mathrm{NH}_{4}-\mathrm{N}$ & $-0,687$ & 0,239 & $-0,430$ & 0,923 & $-2,867$ & 0,008 \\
\hline $\mathrm{N}_{\mathrm{TOT}^{-}}{ }^{\mathrm{N}}$ & 1,384 & 0,454 & 0,456 & 0,923 & 3,045 & 0,006 \\
\hline
\end{tabular}


Tabla 3A) y la concentración de fósforo reactivo soluble (coeficiente estandarizado $=-0,547$; Tabla $3 \mathrm{~A}$ ) mostraron un patrón inverso con la abundancia total de fitoplancton, la conductividad específica se asoció en forma positiva (coeficiente estandarizado $=0,424$; Tabla 3A).

Las variables predictoras en las estaciones El Muro y Alhué fueron la concentración de nutrientes nitrogenados orgánicos e inorgánicos (Tablas 3B y $3 C)$. La concentración de nitrógeno orgánico $\left(\mathrm{N}_{\mathrm{TOT}}{ }^{-}\right.$ N) fue una variable común a ambas estaciones, sin embargo, los compuestos inorgánicos mostraron un comportamiento diferencial entre ellas. En la estación Alhué, la concentración de nitrato $\left(\mathrm{NO}_{3}-\right.$ $\mathrm{N})$ fue un predictor significativo de la abundancia total de fitoplancton, cuyo poder explicativo fue similar al presentado por el $\mathrm{N}_{\text {TOT }}-\mathrm{N}$ (Tabla 3B). Por el contrario, en la estación El Muro, la concentración de amonio $\left(\mathrm{NH}_{4}-\mathrm{N}\right)$ fue una variable relevante, con un peso similar al presentado por el $\mathrm{N}_{\text {TOT }}-\mathrm{N}$ para explicar el patrón de abundancia fitoplanctónica (Tabla 3C). A diferencia del nitrógeno orgánico, los nutrientes inorgánicos se asociaron en forma inversa con la abundancia de fitoplancton $\left(\mathrm{NO}_{3}-\mathrm{N}=-0,448 ; \mathrm{NH}_{4}-\mathrm{N}=-0,430\right.$; Tablas 3B y 3C).

\section{DISCUSIÓN}

A pesar que la abundancia total de fitoplancton en los sectores Las Balsas y El Muro se agrupó en forma significativa, diferencias de variables predictoras entre estas estaciones sugieren mecanismos alternativos de regulación de la abundancia que estarían asociados a la estabilidad de la columna de agua. Las Balsas representa un sector próximo a la descarga de los principales tributarios, los ríos Cachapoal y Tinguiririca. La intensidad y frecuencia de descarga de estos cursos de agua produce eventos localizados de turbulencia y la generación de procesos advectivos en la columna de agua (Hillmer \& Niño 2000'1). Bajo esta condición de inestabilidad, los patrones de abundancia de fitoplancton estarían siendo regulados principalmente por factores físicos, lo cual ha sido sugerido por otros autores para este sector del embalse Rapel (Soto et al. 1984, Reynolds et al. 1986, Contreras et al. 1994, Lavanderos et al. 1994, Niño et al. $2001^{2}$ ), así como para sectores

${ }^{2}$ NIÑO Y, A DE LA FUENTE, G MARTÍNEZ, M CONTRERAS \& I VILA (2001) Evolución del fitoplancton en el embalse Rapel. Modelación de las interacciones entre los sistemas físico y biológico. Memorias del XIV Congreso de Ingeniería Sanitaria y Ambiental Aidis-Chile, Santiago, Chile. análogos en otros embalses (Henry et al. 1998, Berrada et al. 2000, Han et al. 2000, Kwang-Guk \& Jones 2000). La estrecha asociación con la temperatura y la conductividad específica en el sector Las Balsas apoyaría la propuesta de una regulación externa de la abundancia de fitoplancton modulada por factores físicos. Además dadas las condiciones de turbulencia y de oxigenación de la columna en el sector Las Balsas, es altamente probable que la única fuente de fósforo reactivo soluble provenga desde la cuenca de avenamiento, cuya disponibilidad esté asociada al patrón estacional de escurrimiento, confirmando con ello el control externo antes mencionado. También, es predecible que durante los periodos de estiaje (verano-otoño) disminuya la concentración de este nutriente en el embalse y genere una marcada alternancia temporal en la biota del sistema.

Un escenario altamente diferente puede plantearse en las estaciones El Muro y Alhué. En ambos sectores los compuestos nitrogenados fueron relevantes en explicar los patrones de distribución de fitoplancton, lo que podría explicarse por la absorción de nutrientes por algas. La asociación entre la abundancia de fitoplancton y nutrientes nitrogenados en estos sectores surgiría como consecuencia de condiciones hidrodinámicas localizadas, dado por una mayor estabilidad física de la columna de agua en la estación El Muro y un aislamiento hidrodinámico en la estación Alhué.

En el sector El Muro se ha descrito un proceso de estratificación y eventos periódicos de anoxia en el hipolimnion (Vila et al. 2000). Bajo estas condiciones reductoras, la forma predominante de nitrógeno inorgánico es el amonio, el que se estaría generando por la mineralización de materia orgánica en el sedimento, tornándose el amonio en la principal fuente de nutrientes nitrogenados en este sector del embalse. Esta asociación con amonio sugiere un control predominantemente interno sobre la abundancia de fitoplancton durante el periodo estival. Sin embargo, es altamente probable que la homogeneidad térmica observada en otros periodos del año produzca un menor aporte interno de amonio y su predominio disminuya en el periodo invernal de crecidas, cuando la importación de compuestos nitrogenados y de materia orgánica desde la cuenca de avenamiento adquiere mayor relevancia (Niño et al. $2001^{2}$ ).

El sector Alhué presenta un régimen de circulación y condiciones batimétricas que propician prácticamente su aislamiento hidrodinámico (Contreras et al. 1994, Hillmer \& Niño 2000¹). La condición somera y el alto grado de exposición a vientos predominantes generan un proceso 
advectivo en toda la columna de agua y un alto potencial de oxidación a través del año. Bajo este escenario, se promueven las formas nitrogenadas oxidadas cuya disponibilidad es independiente de la época del año. Aunado a lo anterior, es probable que el régimen de circulación promueva un mayor tiempo de residencia hidráulico en este sector, lo cual gatillaría el predominio de reacciones internas en la cubeta. Además, se ha descrito un significativo proceso de descomposición de materia orgánica en forma periódica en el sector Alhué, lo que estaría generando la difusión de fósforo reactivo soluble, metano $\left(\mathrm{CH}_{4}\right)$ y dióxido de carbono $\left(\mathrm{CO}_{2}\right)$ desde el sedimento hacia la

\section{(A) Subunidad Lis Balsns}

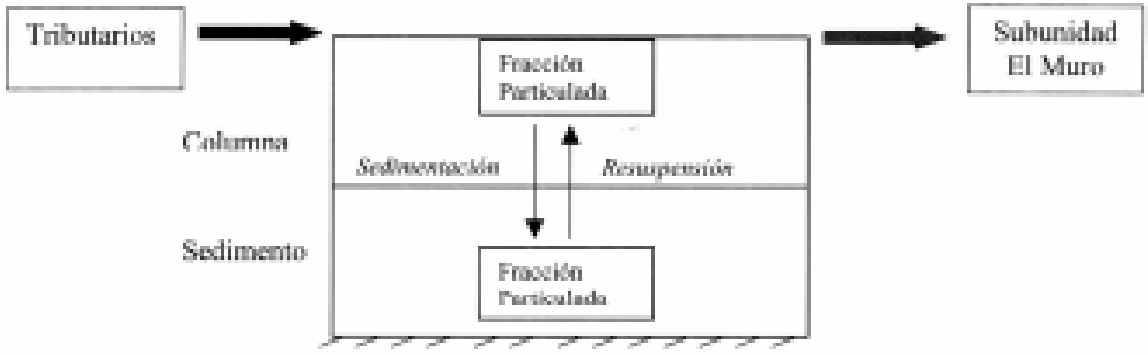

(B) Subunidad E1 Muro

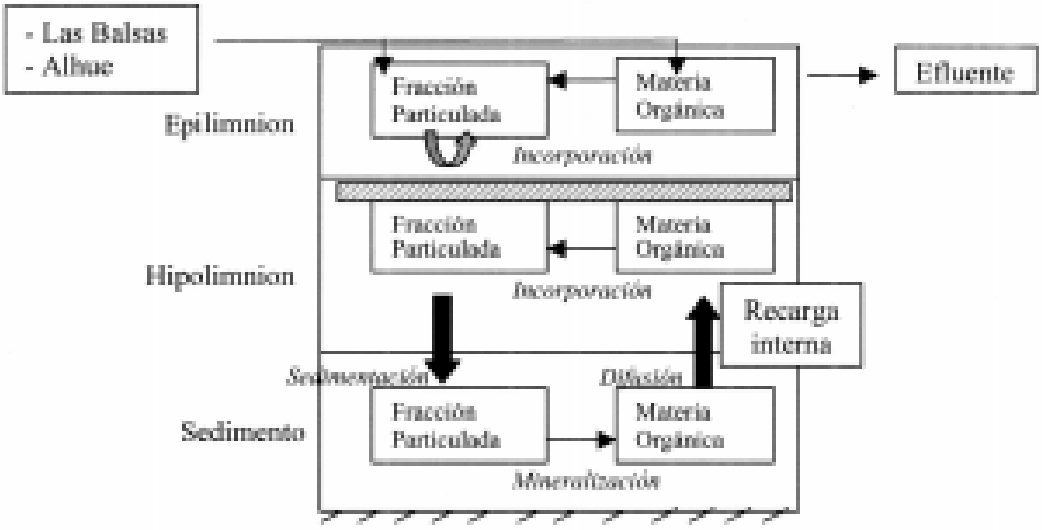

(C) Subunidad Albue

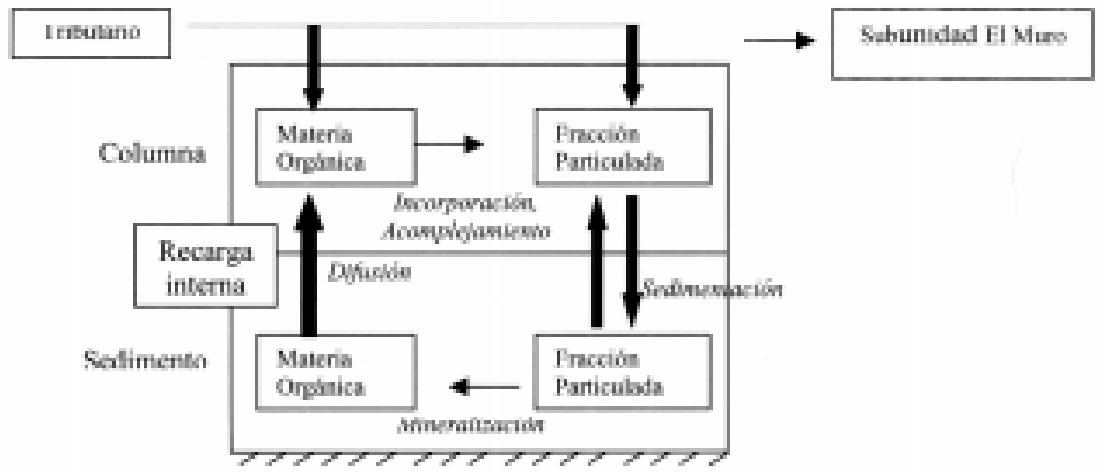

Fig. 4: Modelos conceptuales de la abundancia total de fitoplancton en tres sectores del Embalse Rapel. Cada esquema resume los componentes de cada sistema definido por compartimentos en la columna de agua y mecanismos físicos y químicos asociados a particulas de fitoplancton y a la materia orgánica proveniente de la mineralización de células.

Conceptual models of phytoplankton total abundance in three sectors at the Rapel reservoir. Each diagram summarizes every components of the system defined by compartments in the water column and physical and chemical mechanisms associated both the phytoplankton particles and the organic matter originated by the cells mineralisation. 
columna de agua (Vila et al. 2000). Este proceso de recarga interna de fósforo, en conjunto con vertidos periódicos de compuestos orgánicos desde la cuenca, estarían generando la actual condición eutrófica que se observa en el sector Alhué (Vila et al. 1997). La doble fuente de compuestos fosforados mantendría en forma permanente una alta disponibilidad de nutrientes en la columna y conduciría a un control por compuestos nitrogenados por sobre los fosforados. Estos antecedentes son coincidentes con la asociación a $\mathrm{NO}_{3}-\mathrm{N}$ y $\mathrm{N}_{\text {Tот }}-\mathrm{N}$ encontrada en este estudio. $\mathrm{Si}$ bien, la estrecha dependencia por $\mathrm{NO}_{3}-\mathrm{N}$ permitiría postular un control externo y regulado por las condiciones hidrológicas de la cuenca, la presencia de compuestos nitrogenados orgánicos $\left(\mathrm{N}_{\text {тот }}{ }^{-}\right.$ $\mathrm{N})$ estaría indicando también un no despreciable control interno de la abundancia de fitoplancton, asociado a la condición léntica del sector Alhué.

Nuestros resultados muestran la existencia de al menos tres compartimentos físicos en el Embalse Rapel, con diferentes factores y mecanismos de regulación. Para integrar esta información, se postulan tres modelos conceptuales de abundancia total de fitoplancton, asociados a los tres sectores del embalse.

En el sector Las Balsas se propone un modelo de funcionamiento en el que predominaría un control externo, proveniente desde los tributarios, con una marcada unidireccionalidad en el régimen de circulación (Fig. 4A). El modelo presenta dos estratos: columna de agua y sedimento, entre los que operarían los procesos de sedimentación y resuspensión de partículas de fitoplancton (Fig. 4A). A las variables forzantes determinadas en este sector se adiciona la concentración de sólidos totales en base a los numerosos antecedentes que muestran la influencia de esta variable en los patrones de abundancia y producción de fitoplancton (Cabrera et al. 1977, Montecino \& Cabrera 1982, Reynolds et al. 1986, Lavanderos et al. 1994).

Por el contrario, para el sector El Muro se postula un modelo con un control predominantemente interno (Fig. 4B). Este modelo incorpora una condición de estratificación a través de la implementación de dos estratos en la columna de agua: epilimnion e hipolimnio, entre los que no habría un intercambio de componentes. Los procesos que se establecen entre el sedimento y el hipolimnion son la sedimentación y resuspensión de partículas, así como la difusión en ambas direcciones de la materia orgánica proveniente de la mineralización de biomasa (Fig. 4B). En el estrato epilímnico operaría un proceso de incorporación de materia orgánica en la fracción particulada a través de la absorción de nutrientes. También en este estrato se incluye el aporte tem- poral de nutrientes nitrogenados $\left(\mathrm{NO}_{3}-\mathrm{N}\right)$ desde el sector Las Balsas (Fig. 4B).

Para el sector Alhué se propone un modelo basado en un origen doble en el control de la abundancia total fitoplanctónica. El control operaría a través de los aportes de nitrógeno orgánico e inorgánico desde los tributarios y por la generación interna de nutrientes orgánicos (Fig. 4C). Se incluyen los estratos columna de agua y sedimento, entre los que se establecen los procesos físicos de sedimentación y resuspensión de partículas, así como los procesos químicos adsorción y desorción por ligandos orgánicos.

Desde su creación hasta la actualidad, se ha desarrollado una intensa investigación en la cuenca del Embalse Rapel, en la que inicialmente aparece la descripción de numerosos patrones de distribución. A pesar que estos estudios sugieren fuertemente que una condición de heterogeneidad espacial estaría modulando patrones y procesos en este embalse, la ausencia de una aproximación sistémica y el supuesto de independencia espacial entre los diferentes sectores, ha impedido reconocer algunos de los principales mecanismos asociados a estos patrones. Por lo tanto, como una extensión de los estudios empíricos largamente desarrollados en el Embalse Rapel, en este estudio se recopila e integra información que permite proponer modelos conceptuales de funcionamiento, cuyo desarrollo matemático e implementación práctica pudieran explicar los complejos patrones de distribución de fitoplancton que se desarrollan en sistemas espacialmente heterogéneos.

\section{AGRADECIMIENTOS}

Este estudio fue financiado por el proyecto de investigación FONDECYT 1010483, Universidad de Chile. Los autores agradecen a Yerko Niño por su asesoría en numerosos aspectos teóricos del estudio.

\section{LITERATURA CITADA}

ANTENUCCI J (1996) Hidrobiological modelling of Rapel reservoir, Central Chile. Honours Thesis, Department of Environmental of Engineering, University of Western Australia, Crawley, Australia. xxi + 115 pp. ARMENGOL J, JC GARCÍA, M COMERMA, M ROMERO, J DOLZ, M ROURA, BH HAN, A VIDAL \& K SIMEK (1999) Longitudinal processes in canyon type reservoirs: the case of Sau (N.E. Spain). En: Tundisi JG \& M Straskraba (eds) Theoretical reservoir ecology and its applications: 313-345. International Institute of Ecology, Brazilian Academy of Science and Backhuys Publishers, São Carlos, Brazil. 
APHA (1995) Standards methods for the examination of water and wastewater, AWWA, WEF, APHA. Nineteenth edition. AWWA, WEF, APHA, Washington, District of Columbia, USA. xxx + 1.008 pp.

BAHAMONDE N \& S CABRERA (1984) Embalses, fotosintesis y produtividad primaria. Alafabeta Impresores, Universidad de Chile, Santiago, Chile. 234 pp.

BERRADA D, R BERRADA, A BENZEKRI \& A FAHDE (2000) Horizontal heterogeneity of microphytoplanktonic and zooplanktonic communities in relation to abiotic parameters in the lake-reservoir El Kansera (Morocco). Revue des Sciences de 1'Eau 13: 213-236.

CABRERA S, V MONTECINO, I VILA, N BAHAMONDE, I BAHAMONDE, I BARENDS, R RODRÍGUEZ, R RUIZ \& D SOTO (1977) Características limnológicas del embalse Rapel, Chile central. Organización de Estados Americanos, Departamento de Asuntos Científicos y Tecnológicos, Monografía 1: 40-61.

CONTRERAS M (1992) Desplazamientos diarios de Odontesthes bonariensis (Valenciennes) en el embalse Rapel (Pisces: Atherinidae). Tesis de Magíster en Ciencias Biológicas, Facultad de Ciencias, Universidad de Chile, Santiago, Chile. 82 pp.

CONTRERAS M, H VILLAGRÁN \& C SALAZAR (1994) Características hidrodinámicas del embalse Rapel. Medio Ambiente (Chile) 12: 41-49.

ESPÍNDOLA ELG, T MATSUMURA-TUNDISI, AC RIETZLER \& JG TUNDISI (2000) Spatial heterogeneity of the Tucurui reservoir (State of Parana, Amazonia, Brazil) and the distribution of zooplanktonic species. Revista Brasilera de Biología 60: 179-194.

FERNÁNDEZ-ROSADO MJ, J LUCENA \& F NIELL (1994) Space-time heterogeneity of the chlorophylla distribution in La Concepción reservoir (Istan, Málaga): representative models. Archives für Hydrobiologie 129: 311-325.

GUREVITCH J \& ST CHESTER JR (1986) Analysis of repeated measures experiments. Ecology 67: 251255.

HAN BO-PING, J ARMENGOL, JC GARCÍA, M COMERMA, M ROURA, J DOLZ \& M STRASKRABA (2000) The thermal structure of Sau Reservoir (NE: Spain): a simulation approach. Ecological Modelling 125: 109-122.

HAIR JF JR, RE ANDERSON, LT TATHAM \& WC BLACK (1995) Multivariate data analysis. Fourth edition. Prentice Hall, Upper Saddle River, New Jersey, USA. 745 pp.

HENRY R, MA NUNES, PM MITSUKA, N LIMA \& SMC CASANOVA (1998) Variação espacial e temporal da productividade primária pelo fitoplancton na Represa de Jurumirim (Río Paranapanema, SP). Revista Brasilera de Biología 58: 571-590.

KENNEDY RH (1999) Reservoir design and operation: limnological implications and management opportunities. En: Tundisi JG \& M Straskraba (eds) Theoretical reservoir ecology and its applications: 128. International Institute of Ecology, Brazilian Academy of Science and Backhuys Publishers, São Carlos, Brazil.
KAWARA O, E YURA, S FUJII \& T MATSUMOTO (1998) A study on the role of hydraulic retention time in eutrophication of the Asahi river dam reservoir. Water Science and Tecnology 37: 245-252.

KWANG A \& JR JONES (2000) Temporal and spatial patterns in salinity and suspended solids in a reservoir influenced by the Asian monsoon. Hydrobiologia 436: 179-189.

LAVANDEROS L, H VILLAGRÁN \& H MÜHLHAUSER (1994) Temporal and spatial changes in primary biomass as a diagnosis and prognosis in environmental impact (Rapel reservoir, central Chile). Environmental Monitoring and Assessment 29: 53-64.

LIND OT \& L DÁVALOS-LIND (1999) Suspended clay: its role in reservoir productivity. En: Tundisi JG \& M Straskraba (eds) Theoretical reservoir ecology and its applications: 85-97. International Institute of Ecology, Brazilian Academy of Science and Backhuys Publishers, São Carlos, Brazil.

MARDIA KV, JT KENT \& JM BIBBY (1995) Multivariate analysis. Academic Press, London, United Kingdom. $518 \mathrm{pp}$.

MONTECINO V \& S CABRERA (1982) Phytoplankton activity and standing crop in an impoundment of central Chile. Journal of Plankton Research 4: 943950.

MÜHLHAUSER HA, L SOTO \& P ZAHRADNIK (1987) Improvement of the Kjeldahl method for total nitrogen including acid-hydrolyzable phosphorus determinations in freshwater ecosystem. International Journal of Environmental Analytical Chemistry 28: 215-226.

NOGUEIRA MG (2000) Phytoplankton composition, dominance and abundance as indicators of environmental compartmentalization in Jurumirim reservoir (Paranapanema river), São Paulo, Brazil. Hydrobiologia 43: 115-128.

PADISÁK J, J KÖHLER \& S HOEG (1999) The effect of changing flushing rates on development of late summer Aphanizomenon and Microcystis populations in a shallow lake, Müggelsee, Berlin, Germany. En: Tundisi JG \& M Straskraba (eds) Theoretical reservoir ecology and its applications: 411-423. International Institute of Ecology, Brazilian Academy of Science and Backhuys Publishers, São Carlos, Brazil.

PÉREZ MC, S BONILLA \& G MARTÍNEZ (1999) Phytoplankton community of a polymictic reservoir, La Plata River basin, Uruguay. Revista Brasilera de Biología 59: 535-541.

POURRIOT R, A TIFNOUTI \& C ROUGIER (1994) Summer spatial distribution of zooplankton in the reservoir Lalla Takerkoust, Morocco. Archives für Hydrobiologie 130: 113-127.

REYNOLDS CS, V MONTECINO, ME GRAF \& S CABRERA (1986) Short-term dynamics of a Melosira population in the plankton of an impoundment in central Chile. Journal of Plankton Research 8: 715-740.

ROMERO JR \& J IMBERGER (1999) Seasonal horizontal gradients of dissolved oxigen in a temperate austral reservoir. En: Tundisi JG \& M Straskraba (eds) Theoretical reservoir ecology and its applications: 211-226. International Institute of Ecology, Brazilian Academy of Science and Backhuys Publishers, São Carlos, Brazil. 
SOTO D, I VILA \& B VILLALOBOS (1984) Temporal and spatial distribution of rotifera in a Chilean reservoir: a possible effect of impoundment hydrodynamics. Hydrobiologia 114: 67-74.

STRASKRABA M (1999) Retention time as a key variable of reservoir limnology. En: Tundisi JG \& M Straskraba (eds) Theoretical reservoir ecology and its applications: 385-410. International Institute of Ecology, Brazilian Academy of Science and Backhuys Publishers, São Carlos, Brazil.

THORNTON KW (1990) Perspective on reservoir limnology. En: Thornton KW, BL Kimmel \& FE Payne (eds) Reservoir limnology: ecological perspectives: 1-13. John Wiley and Sons Inc., New York, New York, USA.

TUNDISI JG (1993) Theoretical basis for reservoir management. Verhandlungen Internationale Verein Limnologie 25: 1153-1156.

TUNDISI J, O ROCHA, T MATSUMURA-TUNDISI \& B BRAGA (1998) Reservoir management in South America. Water Resource Development 14: 141-155.

TUNDISI JG, T MATSUMURA-TUNDISI \& O ROCHA (1999) Theoretical basis for reservoir management. En: Tundisi JG \& M Straskraba (eds) Theoretical reservoir ecology and its applications: 505-528. International Institute of Ecology, Brazilian Academy of Science and Backhuys Publishers, São Carlos, Brazil.
VILA I, I B ARENDS \& V MONTECINO (1987) Abundancia y distribución temporal del fitoplancton en el embalse Rapel, Chile central. Revista Chilena de Historia Natural 60: 37-55.

VILA I, M CONTRERAS, V MONTECINO \& J PIZARRO (1997) Eutrophication and phytoplankton selective response in a temperate reservoir. Verhandlungen Internationale Verein Limnologie 26: 798-802.

VILA I, M CONTRERAS, V MONTECINO, J PIZARRO \& DD ADAMS (2000) Rapel: a 30 years temperate reservoir: eutrophication or contamination? Archives für Hydrobiologie 55: 31-44.

WILKINSON L, M HILL, JP WEINA \& GK BIRKENBEUEL (1992) SYSTAT for Windows: Statistics. Fifth edition. SYSTAT Inc., Evanston, Illinois, USA. 750 pp.

ZAHRADNIK P (1981) Methods for freshwater analysis. International Courses for limnology. UNESCO, University of Viena, Wiena, Austria. 43 pp.

Editor Asociado: Vivian Montecino

Recibido el 17 de diciembre de 2001; aceptado el 25 de marzo de 2003 